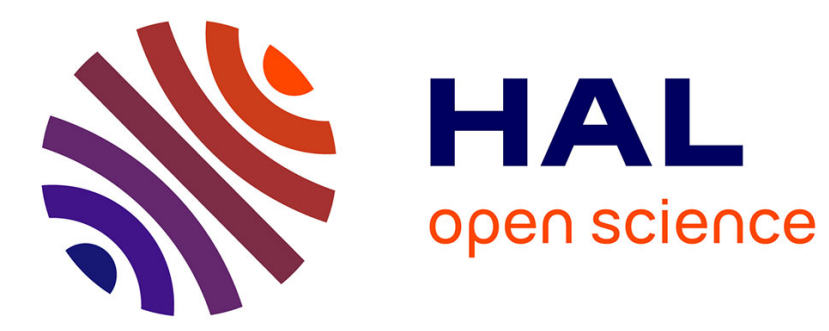

\title{
La représentation de discours indirects non actualisés dans À la recherche du temps perdu
}

Bérengère Moricheau-Airaud

\section{To cite this version:}

Bérengère Moricheau-Airaud. La représentation de discours indirects non actualisés dans À la recherche du temps perdu. L'information grammaticale, 2007, 112 (1), pp.9-15. halshs-01979801

\section{HAL Id: halshs-01979801 https://shs.hal.science/halshs-01979801}

Submitted on 13 Jan 2019

HAL is a multi-disciplinary open access archive for the deposit and dissemination of scientific research documents, whether they are published or not. The documents may come from teaching and research institutions in France or abroad, or from public or private research centers.
L'archive ouverte pluridisciplinaire HAL, est destinée au dépôt et à la diffusion de documents scientifiques de niveau recherche, publiés ou non, émanant des établissements d'enseignement et de recherche français ou étrangers, des laboratoires publics ou privés. 


\section{La représentation de discours indirects non actualisés dans $\dot{A}$ la} recherche du temps perdu

Bérengère Moricheau-Airaud

\section{Citer ce document / Cite this document :}

Moricheau-Airaud Bérengère. La représentation de discours indirects non actualisés dans $\dot{A}$ la recherche du temps perdu. In: L'Information Grammaticale, N. 112, 2007. pp. 9-15.

doi : 10.3406/igram.2007.3865

http://www.persee.fr/doc/igram_0222-9838_2007_num_112_1_3865

Document généré le 15/12/2015 


\section{LA REPRÉSENTATION DE DISCOURS DIRECTS NON ACTUALISES DANS À LA RECHERCHE DU TEMPS PERDU}

\section{Bérengère MORICHEAU-AIRAUD}

Les terminologies «paroles» ou encore «discours rapporté(e)s »1, qui apparaissent le plus souvent dans les grammaires scolaires actuelles pour désigner les modalités d'articulation des voix du narrateur et des personnages, et plus largement de deux énonciateurs, ne donnent pas une image exacte du champ linguistique de la représentation de l'hétérogénéité discursive au sein d'un dire premier. Si tout fait de représentation de discours autre implique l'association de deux actes d'énonciation, l'un accomplissant l'opération métadiscursive de représentation de l'autre, il importe en effet de ne pas réduire les rapports d'hétérogénéité entre ces deux énonciations à une différence de l'ensemble des paramètres les définissant respectivement - locuteurs, allocutaires, lieu et temps - sous l'influence du caractère prototypique du «il lui a dit » résultant des relations privilégiées entre récit romanesque et analyse linguistique 2 . Même si les analyses à venir porteront sur une œuvre littéraire, on se gardera de cette compression méthodologique ramenant au seul domaine littéraire la réalité d'une pratique quotidienne. En fait, l'hétérogénéité entre deux actes d'énonciation peut relever d'une différence dans leur situation respective ne touchant qu'à un seul paramètre, dans le cas par exemple de représentation d'un dire de soi autre uniquement lié à un contraste en termes de temporalité - selon le modèle : «Je t'ai moi-même dit hier : "N'oublie pas la lettre!" ». Discours autre et discours d'un autre ne sont donc pas assimilables, ce qui va sensiblement à l'encontre des expressions les plus courantes citées plus haut dont on constate là qu'elles laissent de côté des cas qui relèvent pourtant pleinement du champ de la représentation de discours autre, tels que « II dira : "[...]". », « Dis-lui donc : "[...]". », « II aurait dû lui dire : "[...]". ». De fait, et c'est ce qui nous intéresse là, représenter n'est pas forcément rapporter : le dire autre n'est pas forcément un dire antérieur ayant déjà été actualisé au sein de la fiction.

Les analyses de Jacqueline Authier-Revuz, qui propose et justifie pleinement le passage de la dénomination «paroles rapportées » à «représentation de discours autre », se montrent de fait très vigilantes - et donc très pertinentes -

1. Désormais, les différentes configurations de représentation de dire autre seront abrégées en DD, DI, DIL par exemple.

2. «[O]n ne saurait enfermer dans la visée particulière d'un genre littéraire l'approche du fait langagier de base de l'hétérogénéité discursive et de ses formes - formes de présentation du discours autre dans le discours par lesquelles celle-ci se manifeste. », Tomassone (2001: 198). quant à ces points de frontières du champ de la représentation de discours autre :

Pour le DD : on a vu qu'il ne fallait pas confondre autonymie, ou mention, avec textualité du rapport d'un [message] produit antérieurement, puisque le DD peut produire ces actes d'énonciation n'ayant pas encore eu lieu, ou ne devant jamais avoir lieu 3.

Cette dernière distinction touchant à la représentation de discours dont la non-actualisation constitue l'hétérogénéité est fondamentale dans une énonciation telle que la narration de $\dot{A}$ la Recherche du Temps perdu au moins pour deux raisons; d'une part la configuration de DD y est majoritaire de manière écrasante pour la représentation de discours autre qui en constitue plus généralement un fait textuel conséquent - avec seulement un fléchissement sensible de cette présence dominante au profit du DI dans des tomes tels que La Prisonnière et Albertine disparue dans lesquels les scènes mondaines sont moins nombreuses et le héros davantage replié sur lui-même ${ }^{4}$; d'autre part la représentation de discours qui n'ont pas été actualisés - d'un point de vue interne à la fiction puisque réellement aucun ne l'a été - est un phénomène conséquent et qui touche d'autres configurations de représentations de discours autres, notamment le $\mathrm{DI}$ - et à cet égard les remarques à venir pourront être reconduites pour cet autre corpus. Ainsi, les représentations d'actes d'énonciations autres non actualisés émergent notablement comme une des variations du fait langagier et en l'occurrence textuel de monstration d'hétérogénéité discursive qui apparaît dès lors comme travaillé d'une dynamique interne quant à ses diverses réalisations - par exemple quand ceci fait ressentir comme plus dramatiques les DD bel et bien actualisés, par exemple ceux d'Albertine avant sa disparition.

Quoi qu'il en soit, les caractéristiques de la configuration de DD restent identiques (que l'acte d'énonciation autre ait été actualisé ou non), ce que la proximité fréquente de deux occurrences de DD, l'un actualisé et l'autre non, fait ressortir:

Enfin on demanda les voitures. Mme Verdurin dit à Swann : « Alors, adieu, à bientôt, n'est-ce pas ? » tâchant par l'amabilité du regard

3. (1992-1993: 14). L'ensemble des analyses à venir s'appuiera donc sur ses théories. Leur pertinence se ressent également, par exemple face aux positions de Laurence Rosier, autour de la question de ce que cette dernière désigne comme des «formes hybrides » alors que Jacqueline Authier-Revuz leur reconnaît un statut énonciatif propre.

4. Au-delà de la triade de la vulgate, DD/DI/DIL, les modalisations autonymiques interdiscursives interprétatives telles qu'elles sont définies par J. Authier-Revuz (1992-1993 : 41) sont également très nombreuses. 
et la contrainte du sourire de l'empêcher de penser qu'elle ne lui disait pas, comme elle l'eût toujours fait jusqu'ici : « À demain à Chatou, à après-demain chez moi. » 5

Les deux DD de cet extrait proustien suivent en effet les mêmes modalités de représentation de dire autre, au-delà de la répétition frappante du même couple énonciatif $\mathrm{Mme}$ Verdurin/Swann. De fait, dans les deux occurrences, on observe bien que l'énonciateur premier, le narrateur, rapporte sur le fil de son propre dire un acte d'énonciation autre dont l'énonciateur second est Mme Verdurin. L'ensemble des DD se compose alors à chaque fois d'une structure bipartite marquée avec, d'une part, dans les segments d'introduction du dire second, la présentation par le narrateur avec ses mots en usage de l'acte représenté dont il fournit une description (et l'on constate là la répétition comme souvent dans la narration proustienne du verbe «dire ", ainsi que la précision du locuteur rapporté «Mme Verdurin » puis "elle», et de l'allocutaire rapporté, "Swann » puis « lui »), et d'autre part, isolés sur le fil de la narration par la forme suprasegmentale des guillemets, la mention des mots des messages représentés (en autonymie dans la partie montrée), respectivement «Alors, adieu, à bientôt, n'est-ce pas? » et «À demain à Chatou, à après-demain chez moi » : les caractéristiques des deux configurations de DD sont identiques, semblables en cela aux modalités suivies pour cette représentation dans l'ensemble de la Recherche. Pourtant, le premier des deux DD représente un dire autre qui a bien été tenu - dans l'univers fictionnel - par Mme Verdurin, comme le souligne l'aspect limitatif du passé simple, alors que le second n'a pas été réalisé lors des adieux, ce que la négation autour du verbe «ne lui disait pas", ainsi que le subjonctif plus-que-parfait marqueur d'un irréel du passé traduisent - et de même les parallélismes antithétiques entre l'irrémédiable de «adieu » et la proximité de «À demain », la précision aimable du "après-demain » et le flou peu pressant du «à bientôt " concrétisent ce contraste. En d'autres termes, l'actualisation ou la non-actualisation de DD constituent des variations internes à cette structure de représentation de dire autre dont le champ se révèle ainsi particulièrement dynamique. Et pour devancer un peu l'interprétation de l'effet produit sur le lecteur par cette représentation d'un discours autre non actualisé, surtout à proximité d'un premier qui, lui, l'a été, on peut au moins constater que la présence d'un dire virtuel rend la situation encore plus dure, et $\mathrm{Mme}$ Verdurin apparaît comme encore plus cruelle, que dans le cas de l'absence de ce qui aurait pu être dit; en allant encore plus loin, c'est même la co-présence, et de là la tension qui naît de celle-ci, entre les deux DD qui les fait percevoir de manière antithétique comme plus douloureux et plus appréciable; de la dynamique linguistique qui se fait tension aux yeux de l'interprétation, se dégage l'ironie critique du narrateur face à Mme Verdurin.

Par ailleurs, le DD non actualisé peut apparaître en dehors de toute proximité d'une autre représentation de dire autre, comme dans :

Mais je lui répétais : «Viens me dire bonsoir », terrifié en voyant que le reflet de la bougie de mon père s'élevait déjà sur le mur,

5. Du Côté de chez Swann, Paris, Gallimard, Folio Classique, p. 280. mais aussi usant de son approche comme d'un moyen de chantage et espérant que maman, pour éviter que mon père me trouvât encore là si elle continuait à refuser, allait me dire : "Rentre dans ta chambre, je vais venir. » 6

Cette fois, c'est la seule tournure périphrastique formée du semi-auxiliaire «aller » et de l'infinitif « dire ", ancrant le dire autre dans le futur imminent, qui invite le lecteur à vivre la scène comme si tout se déroulait sous ses yeux alors qu'il s'agit de toute façon non seulement d'une fiction mais surtout d'un récit rétrospectif.

\section{LES MARQUES LINGUISTIQUES DE LA NON ACTUALISATION DES DD}

Si les deux passages cités ci-dessus ont déjà fait apparaître que la non actualisation pouvait être marquée aussi bien sur le plan lexical - avec les négations - que sur celui de la grammaire - avec les aspects virtuels du subjonctif et inchoatif de la tournure "allait me dire", un recensement des diverses marques de la non-actualisation de DD demande à être dressé à partir des occurrences de DD non réalisés relevés dans les différents tomes de la Recherche.

\subsection{Les marques grammaticales: les temps et les modes des verbes introducteurs}

Ce qui marque le plus souvent la virtualité des DD, c'est le plus souvent leur arrivée après un verbe de parole dans le segment introducteur conjugué à un mode induisant une certaine virtualité ou à un temps signalant la postériorité de la réalisation du procès d'énonciation.

\subsubsection{Systèmes hypothétiques complets}

La marque la plus fréquente de la non actualisation du DD est son intégration dans un système hypothétique, la plupart du temps complet, c'est-à-dire que l'on retrouve le jeu de deux propositions, l'une subordonnée conjonctive hypothétique et l'autre principale répondant à la condition.

Bien des fois il eût pu se faire qu'Andrée devant venir me voir à Balbec, si j'avais mensongèrement préparé de lui dire, pour ne pas avoir l'air de tenir à elle : «Hélas, si seulement vous étiez venue il y a quelques jours, maintenant j'en aime une autre, mais cela ne fait rien, vous pourrez me consoler », un peu avant la visite d'Andrée, Albertine me manquait de parole, mon cœur ne cessait plus de battre, je croyais ne jamais la revoir et c'était elle que j'aimais 7 .

Le passage offre de fait bien une structure binaire; dans la proposition subordonnée hypothétique introduite par la conjonction «si ", se rencontre le plus-que-parfait de l'indicatif pour le verbe introducteur de la parole représentée, c'està-dire la version accomplie de l'imparfait, «j'avais mensongèrement préparé de lui dire ", et dans la suivante l'imparfait de l'indicatif avec «manquait », « cessait » et « croyait »; cette structure correspond en français à l'irréel du présent latin, c'est-à-dire à une configuration dans laquelle le procès envisagé dans l'apodose n'est plus posé comme réalisable du

6. Ibid., p. 35

7. Albertine disparue, Paris, Gallimard, Folio Classique, p. 87. 
point de vue du locuteur/rapporteur situé dans le présent; au moment où le narrateur met au plus-que-parfait le verbe introducteur des paroles en mention dans la configuration de DD non actualisé, il place sa réalisation sur un plan hypothétique - par opposition au futur plus catégorique pour lequel le procès appartient encore au monde des attentes.

On rencontre également plus souvent des systèmes hypothétiques complets correspondant à un irréel du passé :

Certes si elle avait dit comme une Odette : "C'est bien vrai ce gros mensonge-là? » je ne m'en fusse pas inquiété, car le ridicule même de la formule se fût expliqué par une stupide banalité d'esprit de femme ${ }^{8}$.

Cette fois, si le procès envisagé dans l'apodose et répondant à la condition de la réalisation du DD n'est plus posé comme envisageable, ce n'est plus à partir du présent mais du passé sur lequel se retourne le narrateur. En réalité, c'est cette configuration qui est la plus fréquente dans la narration proustienne avec, pour marquer cet ancrage dans le passé, le recours au subjonctif plus-que-parfait, suivant un niveau de langue supérieur, encore que assez conforme à l'état de la langue au début du Xxe siècle :

Mais alors que, si elle eût retrouvé à Pierrefonds quelque indifférent, elle lui eût dit joyeusement : «Tiens, vous ici! », et lui aurait demandé d'aller la voir à l'hôtel où elle était descendue avec les Verdurin, au contraire si elle l'y rencontrait, lui, Swann, elle serait froissée, elle se dirait qu'elle était suivie, elle l'aimerait moins, peut-être se détournerait-elle avec colère en l'apercevant 9 .

Dans cet extrait, l'irréel du passé se prolonge dans un DI, ce qui invite à l'élargissement de la pertinence de ces observations aux configurations de discours autres en général même si cela n'est remarquable que dans une mesure moindre par rapport au DD puisque cette configuration de représentation en $\mathrm{DI}$ correspond à une reformulation du dire autre dans les mots en usage de l'énonciateur représentant que l'on peut déjà imaginer plus libre d'évoquer des propos encore non tenus.

Par ailleurs, l'ancrage de l'énonciation dans un irréel du présent ou encore du passé peut également se produire en dehors d'une structure binaire; c'est alors le seul verbe de parole, du fait du temps et/ou du mode auxquels il se trouve conjugué, qui devient le marqueur de l'appartenance au monde des possibles du procès d'énonciation seconde; toutefois, l'effet produit à partir de la non actualisation des DD reste le même, les seules différences étant que le résultat soumis à la condition et placé dans l'apodose n'est plus exprimé et que la configuration paraît moins dramatique et plus suspensive.

Par là M. de Guermantes pouvait être dans ses expressions, même quand il voulait parler de la noblesse, tributaire de très petits bourgeois qui auraient dit : " quand on s'appelle le duc de Guermantes », tandis qu'un homme lettré, un Swann, un Legrandin, ne l'eussent pas dit 10 .

Ce n'est pas elle qui, comme la duchesse de Guermantes (laquelle par sa naissance eût dû être, plus que Mme de

8. La Prisonnière, Paris, Gallimard, Folio Classique, p. 15.

9. Du Côté de chez Swann, cf. note 5, p. 288 et 289.

10. Le Côté de Guermantes, Paris, Gallimard, Folio Classique, p. 227.
Cambremer, à l'abri de ce ridicule), eût dit pour ne pas avoir l'air de savoir le nom peu élégant (alors qu'il est maintenant celui d'une femme les plus difficiles à approcher) de Julien de Montchâteau : « une petite madame... Pic de la Mirandole ». 11

L'emploi du conditionnel passé « auraient dit » ou du subjonctif plus-que-parfait «eût dit», que ce soit pour le verbe de parole lui-même comme dans ces deux passages (où l'on constate la fréquence du verbe « dire »), ou pour des verbes modalisant ceux-ci dans le segment introducteur, place ces énonciations secondes, pourtant représentées sur le fil de la narration, dans le monde des possibles ne pouvant toutefois plus être réalisés : il s'agit à chaque fois d'irréels du passé. Et de même que cela a été signalé pour les systèmes hypothétiques complets, l'effet de contraste est légèrement différent lorsque le temps du verbe est un présent du conditionnel, présentant alors les DD comme n'ayant certes pas été actualisés mais pouvant encore l'être dans l'avenir, sur le modèle des configurations latines de potentiel ou d'irréel du présent - c'est-à-dire entre le fait d'envisager l'énonciation du dire autre comme encore possible ou bien comme ne pouvant plus se réaliser :

Un écrivain sans talent n'aurait qu'à dire : «Votez pour moi à l'Académie parce que ma femme va mourir et que je veux lui donner cette dernière joie. " II n'y aurait plus de salon si on était obligé de faire connaissance de tous les mourants. Mon cocher pourrait me faire valoir : "Ma fille est très mal, faites-moi recevoir chez la princesse de Parme. » [...] 12

\subsubsection{Infinitifs}

II s'agit de l'autre grande marque grammaticale, en termes de fréquence après ce qui relève des systèmes hypothétiques complets ou non, de la virtualité du discours autre représenté pourtant en mention dans des occurrences de DD. Si l'on dessine une échelle qui correspond au mouvement de chronogénèse, on trouvera au terme de celle-ci le mode indicatif, en son milieu le subjonctif, et en son début l'infinitif, forme impersonnelle qui correspond à la variante substantive du verbe; de fait, celle-ci, privée de flexions et rejetant sur un autre verbe dont elle est complément les valeurs de temps et d'aspects - et cela n'est pas à négliger quand on constate que souvent ces verbes recteurs rejettent eux aussi dans le virtuel l'actualisation du dire autre -, se réduit au contenu sémantique du verbe - un peu comme si ce dernier s'en tenait au plan sémiotique de la langue sans être passé du côté sémantique du discours -, c'est-àdire en l'occurrence à la conception sémiotique d'un acte d'énonciation prêt à l'emploi ; or quand les propos sont précisément introduits par un verbe à l'infinitif coupé de toute actualisation, les paroles sont de même perçues comme éloignées de toute réalisation et en revanche placées du côté d'une virtualité... certes déclinable à l'envi mais qui pour l'instant reste comme prête à l'emploi :

De ces chagrins dont elle lui parlait autrefois et qu'il la voyait, sans qu'il fût atteint par eux, entraîner en souriant dans son cours sinueux et rapide, de ses chagrins qui maintenant étaient devenus les siens sans qu'il eût l'espérance d'en être jamais délivré,

11. Sodome et Gomorrhe, Paris, Gallimard, Folio Classique, p. 308. 12. Ibid., p. 80. 
elle semblait lui dire comme jadis de son bonheur : «Qu'est-ce, cela? tout cela n'est rien. » 13

Dans cet extrait, au-delà de l'infinitif « dire », on note de fait encore une modalisation quant aux chances, probables seulement, de réalisation de l'acte d'énonciation rapporté avec le verbe recteur «semblait», ce qui accroît encore le fait que ce procès ne soit alors donné que comme virtuel. En revanche, dans le passage suivant, c'est la référence à l'attitude classique de la cocotte, c'est-à-dire à un dire conventionnel et cliché prêt à l'emploi dans la circonstance en question - donnant une fois de plus une image de la pression qu'exerce l'hétérogénéité constitutive du déjà dit sur l'énonciation du sujet 14 -, qui justifie le caractère virtuel, coupé de tout temps et tensif du temps du procès :

Souriant, elle haussa les épaules, comme pour dire «vous êtes fou, vous voyez bien que ça me plaît ». 15

De manière métaphorique, on pourrait dire que ces DD dont les verbes introducteurs sont à l'infinitif, laissant aux verbes les régissant la charge de marquer temps et aspect, sont eux aussi « à l'infinitif », à une forme «substantive », de base, c'est-à-dire détachés de toute énonciation.

\subsubsection{Futurs}

Enfin, pour être exhaustive quant à ces marques qui ancrent dans le monde des possibles les représentations de discours autre, il faut encore citer le futur, même si celui-ci se rencontre moins souvent que les configurations à l'infinitif et que surtout les irréels au conditionnel et au subjonctif - ce qui n'est guère étonnant dans une œuvre tournée vers le temps perdu, c'està-dire passé :

«Tout ceci, dira le lecteur, ne nous apprend rien sur le manque de complaisance de cette dame; mais puisque vous vous êtes si longtemps arrêté, laissez-moi, monsieur l'auteur, vous faire perdre une minute de plus pour vous dire qu'il est fâcheux que, jeune comme vous l'étiez (ou comme était votre héros s'il n'est pas vous), vous eussiez déjà si peu de mémoire, que de ne pouvoir vous rappeler le nom d'une dame que vous connaissez fort bien. » 16

Qui plus est, pour ce qui est des rapports entre conditionnel et futur, on notera que ce passage est paradoxal dans la mesure où le lecteur n'aura jamais accès à la parole au beau milieu de la narration, qu'à ce titre il équivaut là à un conditionnel, et que son emploi pour le moins surprenant relève d'une pure mise en scène laissant accroire au lecteur en un certain pouvoir alors que c'est bien le narrateur qui tire les ficelles.

\subsection{Les marques lexicales}

L'autre ensemble de marques qui pointent le DD à venir comme n'ayant pas encore été relèvent par ailleurs du lexique.

\subsubsection{Les négations}

Au-delà des indices correspondant à un jeu sur les temps et les aspects, des adverbes de négation, organisés classiquement en couple la plupart du temps autour du verbe de parole ou de celui qui le régit, maintiennent également dans la virtualité les discours autres qu'ils introduisent.

Albertine ne me dit pas plus, à partir de cette soirée, qu'elle n'avait fait dans le passé : «Je sais que vous n'avez pas confiance en moi, je vais essayer de dissiper vos soupçons. » 17

Comme équivalent à cette négation, on rencontre encore des formules de rejet, comme «au lieu de » :

La conversation était rapportée, comme nous l'avons fait, avec la différence qu'au lieu de dire : «M. de Norpois demanda finement », on lisait : " dit avec ce fin et charmant sourire qu'on lui connaît ». 18

Cette expression induit par ailleurs un balancement binaire dans lequel un des dires autres représentés n'est présent qu'en contraste avec l'autre qui a bel et bien été actualisé. Dans l'extrait suivant, le premier DD est posé comme non réalisé au profit du second :

Aussi je n'écoutais jamais ses excuses sans une mauvaise humeur fort injuste et à laquelle venait mettre le comble la manière dont Françoise disait non pas : «J'ai été voir mon frère, j'ai été voir ma nièce ", mais : "J'ai été voir le frère, je suis entrée "en courant" donner le bonjour à la nièce (ou à ma nièce la bouchère) $» .19$

Du reste, certaines occurrences de DD non actualisées cumulent les marques de virtualité, ajoutant les négations à un mode de l'hypothétique :

De Combray au contraire, consultée, elle ne m'eût pas répondu par un triste : «Fais ce que tu veux, tu es assez grand pour savoir ce que tu dois faire ", mais se reprochant de m'avoir laissé seul à Paris, et jugeant mon chagrin d'après le sien, elle eût souhaité pour lui des distractions qu'elle se fût refusées à elle-même [...]. 20

\subsubsection{Les modalisations}

Une autre voie, lexicale, que peut emprunter la non actualisation est celle des modalisations produites par des semiauxiliaires accompagnant les verbes de parole du segment introducteur. En évaluant les chances de réalisation du dire autre représenté comme plus ou moins grandes, ils ancrent celui-ci en même temps qu'il reste dans le plan virtuel. Ainsi, dans :

«Ce n'était pas moi, dut-elle se dire, j'étais aliénée. Moi, je peux encore prier pour mon père ne pas désespérer de sa bonté. » 21

le verbe «devoir » a beau indiquer un degré de probabilité, avec donc des chances élevées pour que ce discours autre (déjà et de toute façon représenté) ait été réalisé, cela signifie en même temps qu'il ne l'a pas été.
13. Du Côté de chez Swann, cf. note 5, p. 342.

14. Cela souligne encore un peu plus la pertinence des appuis théoriques de J. Authier-Revuz.

15. Du Côté de chez Swann, cf. note 5, p. 229

16. Sodome et Gomorrhe, cf. note 11, p. 51.
17. La Prisonnière, cf. note 8 , p. 350 .

18. Albertine disparue, cf. note 7, p. 216.

19. Le Côté de Guermantes, cf. note 10, p. 140.

20. Ibid., p. 336.

21. La Prisonnière, cf. note 8, p. 250. 
De même, on rencontre le même type de non actualisation dans cet extrait avec, cette fois, des chances de réalisation effective nettement moindres :

"Mais vous êtes notre égal, sinon mieux", semblaient, par toutes leurs actions, dire les Guermantes; [...]. 22

Ce verbe est du reste intéressant en ce qu'il rattache la représentation de l'acte d'énonciation second à une traduction, par le narrateur, des actions des Guermantes en dire autre: le narrateur représentant fait parler, de façon littérale, les faits et gestes de ses personnages en DD qu'il donne à lire. On ne peut s'empêcher ici de penser à la phrase du Temps retrouvé qui pose le travail et la tâche d'un écrivain comme étant ceux d'un traducteur - et d'un traducteur en discours, ce qui ne surprendra pas dans ce roman où les personnages sont avant tout des êtres de parole. Par ailleurs, cela lève le voile - et on y reviendra - sur la toute-puissance démiurgique du narrateur qui laisse alors offert à l'interprétation le travail visible de tissage du texte, même s'il n'y a pas encore d'auto-désignation de celui-ci en train de se faire. D'autres expressions, plus ou moins assimilables à des semi-auxiliaires, rectrices des verbes de parole, signalent à la lecture que le dire autre représenté n'a pas été actualisé pour la seule raison qu'il a été donné comme traduction par le narrateur à une attitude ou à des gestes de personnages - et n'a donc pas été à ce titre réalisé :

La duchesse avait l'air de dire : «Qu'est-ce qu'il a ? il est fou. » $[\ldots]^{23}$

Quand elle s'assit, son dos eut l'air de dire : « Dame, il n'y a pas de falaise ici, vous permettez que je m'asseye tout de même près de vous, comme j'aurais fait à Balbec ? » 24

Il arrive même de trouver des segments introducteurs d'un DD non réalisé encore plus explicites quant au fait qu'ils sont une pure création du narrateur au sein de la réalité (de la fiction certes) :

Et Françoise, après un signe modeste, évasif et ravi dont la signification était à peu près : " Chacun son genre; ici c'est à la simplicité », refermait la fenêtre de peur que maman n'arrivât 25 .

\subsubsection{Les procès de dire contrariés}

Il est enfin d'autres verbes, au sein du corpus de représentation de dire autre, qui bloquent le processus de chronogénèse de l'acte d'énonciation rapporté en un point intermédiaire et donc à un stade virtuel, comme le fait par ailleurs dans la langue le mode subjonctif. Ainsi dans :

On avait envie de lui répondre : «Bien entendu que vous ne le saviez pas puisque vous ne connaissez rien à la chose dont il s'agit; comment pouvez-vous même dire que c'est possible ou pas, vous n'en savez rien? En tout cas, maintenant vous ne pouvez pas dire que vous ne savez pas ce que Charcot a dit à du Boulbon, etc., vous le savez puisque nous vous l'avons dit, et vos "peut-être", vos "c'est possible" ne sont pas de mise puisque c'est certain. [...] puisque c'est certain. » 26

22. Sodome et Gomorrhe, cf. note 11, p. 62.

23. La Prisonnière, cf. note 8, p. 31.

24. Le Côté de Guermantes, cf. note 10, p. 340.

25. Ibid., p. 13.

26. Ibid., p. 291.
Il y a bien longtemps aussi que mon père a cessé de pouvoir dire à maman : «Va avec le petit. »

Mais comme pour faire face à l'hypothèse opposée il n'osait pas laisser ce sourire s'affirmer nettement sur son visage, on y voyait flotter perpétuellement une incertitude où se lisait la question qu'il n'osait pas poser : «Dites-vous cela pour de bon? » 27

Que ce soit l'envie, la capacité ou l'audace, le mouvement qui pousse à la réalisation des DD déjà représentés est contrarié de manière interne, par son insuffisance, ou externe, par la rencontre d'un obstacle incontournable, et l'on ne s'en tient qu'à un stade limité de sa réalisation, certes déjà entamée mais non achevée. Précisément, cette dynamique est contrecarrée pour les premières et dernières occurrences citées ci-dessus par un manque de volonté et pour celle du milieu par l'écoulement inéluctable du temps - ce dont on ne s'étonnera pas dans ce roman. Or, dans ce jeu de dynamique mise en échec que matérialise ce type de représentations de dires autres non réalisés, se retrouve deux des grandes tensions qui tiraillent le narrateur, d'une part l'avancée impitoyable du temps contre lequel le héros cherche une solution, d'autre part cette absence de force qui le pousse à toujours remettre à plus tard ses actions et notamment l'écriture de son roman qui apparaîtra comme la «solution» pour « retrouver le Temps». II est donc très révélateur que ces deux problématiques se retrouvent là, liées dans le roman comme autour du fait textuel observé, à partir de la représentation de DD non actualisés. Ne seraitce qu'en cela, celle-ci appelle à une interprétation dans le cadre plus large de la narration proustienne.

\section{LES INTERPRÉTATIONS DE LA NON ACTUALISATION}

Au-delà d'une contribution à une délimitation pertinente du champ de la représentation de discours autre, ces divers faits de non actualisation se révèlent au confluent de plusieurs problématiques qui traversent la narration proustienne, s'inscrivant ainsi au cœur de systèmes et donc de dynamiques porteurs de sens. En cela, ces faits textuels peuvent à bon droit être considérés comme des «formes-sens ».

\subsection{Du soutien à l'illusion réaliste...}

L'interprétation la plus sensible qui se dégage de ces DD non actualisés naît justement de l'effet que de telles représentations de dire autre ont sur le lecteur. Dans cette narration rétrospective au passé - même si l'on n'oubliera à aucun moment qu'il s'agit d'une fiction et que tout ou partie a été inventé -, ces divers faits de non actualisation sont à chaque fois l'occasion pour le narrateur de faire semblant de remettre en jeu l'histoire, comme si rien n'était décidé et que tout se déroulait au fur et à mesure de la lecture, avec des choix encore possibles (ou non avec les hypothétiques à l'irréel) entre diverses paroles à tenir, et de là à représenter. Même si cela est tout à fait impossible pour les raisons évoquées ci-dessus, de telles rencontres sur le fil de l'énonciation du narrateur nourrissent encore un peu plus l'illusion réaliste dans laquelle toute lecture est plongée à des degrés divers,

27. Du Côté de chez Swann, cf. note 5, p. 36 et p. 197 
ainsi que l'identification naturelle du lecteur au narrateur qui dit «je ». Ces moments du texte proustien en font une esquisse bien avant l'heure des romans dont on est le héros, ce qui n'est pas négligeable dans la séduction qu'il exerce sur nous, récepteurs.

\section{2. ... à la tension avec une textualité qui pourrait se laisser percevoir}

En même temps que cela offre à la lecture un certain suspens face aux possibles de l'histoire, cela conforte le narrateur dans une position de toute-puissance, déjà sensible par ailleurs autour des représentations de dire autre, par exemple et entre autres choses quand il condense en une seule occurrence plusieurs actes d'énonciation sur le principe de l'itératif. Alors qu'un réel est déjà posé comme accompli dans cette histoire - certes fictive - au passé, le narrateur fait un tour de force en maintenant en vis-à-vis (ou bien plus généralement en créant quand il s'agit de voies nouvelles pour l'histoire) de nouveaux possibles qui ne pourront de toute façon jamais être actualisés - dans le récit déjà accompli. Cela souligne aux yeux du lecteur la position de démiurge tout-puissant qu'occupe le narrateur, rôle pour le moins déroutant dans cette diégèse menée à la première personne. De là, naît une certaine tension autour de ces représentations de dire autre qui à la fois nourrissent très sensiblement l'illusion réaliste en faisant semblant de rejouer l'histoire, mais dessinent aussi une tension avec l'illusion d'une textualité se tissant sous nos yeux. Bien sûr, cette dernière illusion n'est pas aussi sensible dans la Recherche que dans le Nouveau roman, et pour tout dire c'est l'interprétation seule qui la met en lumière. Néanmoins, de tels constats expliquent la filiation souvent établie - et à juste titre - entre son œuvre et cet autre courant.

\subsection{La relativisation des dimensions du texte proustien}

Si l'on replace au cœur de l'œuvre proustienne cette observation d'un narrateur tout-puissant qui tisse plusieurs fils de l'histoire autour d'un seul actualisé, on retrouve une des variantes d'un fait textuel par ailleurs constaté sur un autre plan : celui des phrases ramifiées 28 qui se développent autour d'un même début mais dans diverses directions. Plus largement encore, cela constitue une des réalisations de la peinture par touche, ou plutôt par facette pour ne pas glisser trop facilement dans la métaphore impressionniste, à laquelle le narrateur se livre pour décrire ses personnages, comme si la réalité - de la fiction certes mais plus largement de sa vie - ne pouvait se saisir que partiellement et ne naître que du rapprochement, de la mise en tension de ces différents aspects.

De ces dynamiques, de ces systèmes de DD, on peut deviner la suggestion de dimensions parallèles 29 non actualisées gravitant autour de l'univers du personnage, ne serait-

28. Bayard (1996), p. 19.

29. À une échelle supérieure et selon un mouvement prudent de mise en abyme, on pourrait même voir dans ces émergences ponctuelles de DD virtuels d'autres variantes de l'histoire, eu égard à la méthode de travail de Proust qui utilisait des cahiers et des paperoles aux rajouts incessants. ce par exemple que la vie de son amante telle que l'imagine un personnage et notamment un personnage jaloux construisant la vie de sa maîtresse en son absence, en concurrence avec la réalité vécue par celle-ci dans la réalité du roman. II s'agirait là d'une certaine façon d'une exploration des couloirs du temps dont on aurait la matérialisation textuelle avec cette juxtaposition de la réalité de la vie des personnages et de ce qui en est au contraire supposé 30 . Cette image est du reste celle sur laquelle se finit le film Le Temps retrouvé de Raoul Ruiz où l'on voit le personnage déambuler dans des catacombes où il retrouve divers objets à même de faire naître ses souvenirs et qui font penser, métaphoriquement, aux méandres de la mémoire.

Par ailleurs, quand on replace l'œuvre proustienne dans son contexte historique où elle est contemporaine des découvertes autour des théories d'Einstein, sa proximité évidente avec la relativité alors conceptualisée apparaît avec ces DD non actualisés. Les mises en regard de dires autres réalisés ou non prennent d'autant plus de sens que la Recherche aboutit sur la décision de se mettre à écrire, en d'autres termes de construire, voire de rejouer, par la fiction, d'autres dimensions du Temps, bien réel lui. Ainsi, si Isabelle Serça 31 en est venue à parler à partir de ses observations sur les parenthèses proustiennes d'une troisième dimension du texte, on peut voir face au maintien de possibles DD non réalisés la concrétisation de mondes parallèles, existant à côté de ce qui apparaît en retour comme une réalité toute relative, que cela soit compris en termes géographiques avec l'exploration de civilisations coexistant à celle d'où vient le héros - on peut penser là aux découvertes de Gulliver - ou bien en termes temporels avec le vécu en simultané d'événements différents de ceux de la (soi-disant) « vraie vie ». Très concrètement, cela pose la littérature comme le moyen de lutter contre l'écoulement du Temps - et c'est bien le message de la fin du Temps retrouvé qui pose l'écriture comme la voie d'accès à une réalité qui remédie à la perte du Temps : celle de l'art. L'interprétation que l'on peut donner de ces DD non actualisés rejoint donc une des grandes conclusions de la narration proustienne, s'inscrivant en cela au cœur d'une des problématiques majeures de cette œuvre, et font donc système avec l'ensemble des réflexions du narrateur.

\subsection{Un accès inédit à l'intériorité des personnages}

Enfin, au-delà de ce rapport étroit qui se dessine pour l'interprétation entre la représentation de dires autres virtuels et la recherche d'un temps perdu, il apparaît également que ces DD non actualisés et pourtant représentés semblent constituer pour la narration une voie nouvelle pour rendre compte de la conscience des personnages, dans la mesure où l'on a alors ce que ceux-ci auraient pu déclarer et où cela

30. Cette juxtaposition se ferait même l'écho de la mise en regard de l'histoire du roman et de la vraie vie de Proust (hypothèse qui gagne en importance et en pertinence du fait de la dimension autobiographique du récit), le roman se faisant ainsi l'image des virtualités non actualisées et qui ne pourront plus l'être de la vie de Proust. II ne s'agit là bien sûr cependant que d'interprétations qu'aucun élément du texte ne vient appuyer, étant donné la rareté déjà notée par les critiques des aveux de la présence de l'auteur dans l'œuvre. 
revient à une matérialisation inédite de la focalisation interne. Bien sûr, cela pose immédiatement, comme de manière plus globale dans l'œuvre, le paradoxe d'une narration homodiégétique avec un héros qui dit je et qui connaît pourtant jusqu'aux moindres pensées des autres personnages - et ce problème est sensiblement accru dans Un amour de Swann. Néanmoins, il est manifeste qu'avec ces DD, la narration s'ouvre ponctuellement sur l'univers parallèle qui correspond, depuis l'intérieur de chaque personnage, à la vision qu'il a du monde de l'histoire. À ce titre, dans le contexte littéraire de l'époque qui réfléchissait entre autres sur la manière de rendre la conscience des personnages 32 , puisque ces DD apparaissent comme une matérialisation renouvelée de la focalisation interne, ils offrent également à l'interprétation un entrebâillement sur la propre construction de l'histoire par le narrateur tant cela renouvelle l'accès à l'intériorité des autres personnages. Même si la textualité ne montre pas ouvertement son avancée, le caractère inédit de cette pénétration est sensible à l'interprétation. Et cette mise en concurrence des diverses perceptions d'un même monde relativise d'autant le réel - de la fiction certes.

Quantitativement, la présence des DD non actualisés sur l'ensemble des DD de la Recherche, certes non majoritaire, n'est pas négligeable : elle s'impose d'autant plus au sein du système des variantes de cette configuration, dont elle suit les modalités caractéristiques de représentation, tant elle apparaît de manière sensible - pour l'analyse - comme une réalisation particulière, aussi bien dans ses marques de non-actualisation que dans ses effets interprétables. De fait, la diversité des traces de la non réalisation du $D D$, avec ses majeures et ses raretés, constitue ces représentations de dires autres non actualisés en système de variations à l'intérieur d'un autre système, celui des DD virtuels au sein des DD. Bien plus encore, c'est l'inscription de ces derniers au sein de problématiques majeures de l'œuvre, telles notamment que la recherche du Temps perdu, la relativisation du monde perçu et enfin la volonté de rendre une intériorité des personnages, qui en fait une forme-sens au cœur aussi bien de systèmes linguistiques qu'interprétatifs. Cela rappelle à quel point dans un texte, tel notamment que celui de la Recherche, ce n'est pas tant la réalité de la fiction qui occupe le devant de la narration que ce qui gravite autour, univers et perceptions parallèles à cette réalité sur lesquels par exemple des DD non actualisés peuvent entrouvrir; pour cette raison ce n'est plus cette réalité qui s'impose à la lecture mais sa mise en concurrence avec des échos différentiels; ou pour être exacte ce n'est pas tant la co-présence d'un DD virtuel et de la réalité d'un autre DD (voire d'aucun dire autre) qui relativise toute perception mais la tension qui s'instaure entre le dire autre qui a été réalisé (ou non d'ailleurs) et celui qui aurait pu l'être et qui résonne sous la narration.
Il est bien frappant de retrouver ici la description du mécanisme de la mention d'un élément faisant écho sous un autre que Sperber et Wilson 33 avancent comme à l'origine des effets d'ironie, dont on sait par ailleurs qu'il s'agit d'une des analyses les plus souvent établies face à l'œuvre proustienne. Et de fait, si l'on revient face aux DD non réalisés avec cette description de la mention-écho, il apparaît en effet que lorsque les DD sont associés par paires, l'un étant actualisé et l'autre non, une tension entre les deux se dessine, l'un résonnant sous l'autre de manière réciproque, et que même en l'absence de paire, le DD non réalisé laisse tout de même percevoir ce qui aurait été énoncé. L'adéquation parfaite de cette description aux configurations de DD virtuels éclaire avec pertinence les effets d'ironie qui s'en dégagent souvent - et cela resterait à développer dans un travail plus vaste. Encore une fois, cela confirme pour la question des DD non actualisés qui nous occupe ici combien ils sont à la confluence de tensions majeures de l'œuvre proustienne.

\section{Bérengère MORICHEAU-AIRAUD} FORELL, EA 3816

Authier-Revuz J., 1992-1993, «Pour l'agrégation, repères dans le champ du discours rapporté ", L'information grammaticale, $\mathrm{n}^{\circ} 55$ et 56, p. 38 à 42 et 10 à 15.

Bayard P., 1996, Le hors-sujet, Proust et la digression, Paris, Les Éditions de Minuit, coll. Paradoxe.

Serça I., 1998, «La parenthèse, troisième dimension du texte proustien ", in J.-M. Defays, L. Rosier, F. Tillon, A qui appartient la ponctuation?, Paris, Duculot.

Sperber D. et Wilson D., 1978, «Les ironies comme mention », in Poétique, $\mathrm{n}^{\circ} 36$.

Tomassone R. (dir.), 2001, Une langue, le français, Paris, Hachette Éducation.

31. Serça (1998).

32. On pense par exemple à la publication du texte Les lauriers sont coupés de Dujardin.

33. Sperber et Wilson (1978) 